\title{
TOPOLOGICAL GROUPS WITH CO-MONOID STRUCTURES
}

\author{
by ELYAHU KATZ
}

(Received 26 March, 1975; revised 6 January and 28 September, 1976)

1. Introduction. The Eckman-Hilton duality [4] reverses arrows in diagrams, turns products to co-products, and multiplications to co-multiplications, etc. In accordance with this process, $\operatorname{Kan}$ [5] obtained the dual of a monoid structure in the category of groups. In this way, we obtain co-monoid structures on topological groups. The main result of this paper is that for $k_{\omega}$ groups (see $\$ 2$ ), we obtain a one-to-one correspondence between the co-monoid structures, and the free topological bases of the group (\$3), thus obtaining topological analogues of the main results of [5].

In [7] a free topological subgroup of the free product of two $k_{\omega}$ groups was discovered. In this paper it is proved that if $G^{\prime}$ and $G^{\prime \prime}$ are two copies of a free $k_{\omega}$ group, then the diagonal of $G^{\prime} \times G^{\prime \prime}$ can be embedded in $G^{\prime} * G^{\prime \prime}$ to give a free topological basis of a free topological subgroup. This subgroup is used in the proof of the main results, which have as application a description of the Pontryagin algebra of a connected group with a co-monoid structure.

I would like to thank Professor Israel Berstein for helpful conversations and suggestions. I am also grateful to the referee whose detailed comments led to considerable improvement in the exposition of this paper.

2. Preliminaries. This work is done in the category of Hausdorff topological groups (TG). For the convenience of the reader we shall state some basic definitions and results in this category, which will be used later on. We start with a theorem of Kan's about co-monoid structures in the category of groups. The co-monoid structures are essentially those of definition 1 of $\$ 3$, provided we strip off the topologies involved.

TheOREM A (See [5].). Let $G$ be a group. Then, there is a one-to-one correspondence between the free bases of $G$ and its co-monoid structures.

Definition A. (a) A $k_{\omega}$ space is a Hausdorff topological space $X$ with compact subsets $X_{n}$, such that: (i) $X=\bigcup_{n=1}^{\infty} X_{n}$, (ii) $X_{n+1} \supset X_{n}$, and (iii) a subset $A$ of $X$ is closed if and only if $A \cap X_{n}$ is compact for all $n$.

(b) By a $k_{\omega}$ decomposition $X=\bigcup_{n=1}^{\infty} X_{n}$ we mean that $X_{n}$ have properties (i), (ii) and (iii).

(c) A $k_{\omega}$ group is a topological group which is also a $k_{\omega}$ space.

Definition B (See [2].). The topological group $F(X)$ is a free topological group with a basis, a pointed topological space $(X, e)$, if it has the following properties: (i) $X$ is a subspace of $F(X)$, (ii) $X-e$ generates $F(X)$ freely as a group, and $e$ is the identity of $F(X)$, and (iii) for any continuous map $l: X \rightarrow G$ such that $G$ is a topological group and $l(e)$ equals the identity of $G, l$ extends uniquely to a homomorphism of topological groups $L: F(X) \rightarrow G$.

Glasgow Math. J. 18 (1977) 145-152.

K 
THEOREM B (See [6].). Let $X=\bigcup_{n=1}^{\infty} X_{n}$ be a $k_{\omega}$ decomposition of $X$. Then $F(X)$ exists and has the following $k_{\omega}$ decomposition: $F(X)=\bigcup_{n=1}^{\infty} F_{n}\left(X_{n}\right)$, where $F_{n}\left(X_{n}\right)$ consists of all reduced words in elements of $X_{n}$ with length less than $n+1$.

Definition C (See [3].). Let $G_{1}$ and $G_{2}$ be topological groups. Then $G_{1} * G_{2}$ is their free product if it satisfies the following: (i) $G_{1}$ and $G_{2}$ are topological subgroups of $G_{1} * G_{2}$, (ii) the underlying group of $G_{1} * G_{2}$ is the free product of the underlying groups of $G_{1}$ and $G_{2}$, and (iii) if $\gamma_{i}: G_{i} \rightarrow H, i=1,2$, are homomorphisms of topological groups, then they extend uniquely to a homomorphism of topological groups $\Gamma: G_{1} * G_{2} \rightarrow H$.

This definition extends to any finite number of groups. We use this notation "*" also for the free product of groups.

Theorem C. (a) (See [3].) Let $G_{1}, \ldots, G_{n}$ be topological groups. Then $G_{1} * \ldots * G_{n}$ exists.

(b) (See [8].) Let $G_{i}=\bigcup_{j=1}^{\infty} G_{i}^{j}$ be $k_{\omega}$ decompositions for $G_{i}, i=1,2, \ldots, n$. Then $G_{1} * \ldots * G_{n}=\bigcup_{j=1}^{\infty} h\left(\left(G_{1}^{j} \vee \ldots \vee G_{n}^{j}\right)^{j}\right)$ is a $k_{\omega}$ decomposition, where $h$ sends an $n$-tuple to the corresponding reduced word in $G_{1} * \ldots * G_{n}$, and $\vee$ is the disjoint union identifying the identities.

(c) (See [7].) For $G_{1}$ and $G_{2}$ topological groups, $G_{1} \times G_{2}$ is embedded in $G_{1} * G_{2}$ via the map $\left(g_{1}, g_{2}\right) \rightarrow g_{1} g_{2}$.

3. Statement of results. Throughout this paper we use the following notation: Let $G^{\prime}$ and $G^{\prime \prime}$ be copies of a topological group $G$ via $i^{\prime}: G \rightarrow G^{\prime}, i^{\prime \prime}: G \rightarrow G^{\prime \prime}$, and let $j^{\prime}: G \rightarrow G^{\prime} * G^{\prime \prime}$, $j^{\prime \prime}: G \rightarrow G^{\prime} * G^{\prime \prime}$ be the natural inclusions induced by $i^{\prime}, i^{\prime \prime}$, respectively. Denote by $D$ the following set:

$$
D=\left\{g^{\prime} g^{\prime \prime} \in G^{\prime} * G^{\prime \prime} \mid \exists g \in G \quad \text { which satisfies } \quad g^{\prime}=i^{\prime}(g), g^{\prime \prime}=i^{\prime \prime}(g)\right\} .
$$

THEOREM 1. Consider $G^{\prime}$ and $G^{\prime \prime}$ with the topologies stripped off, and suppose they are free. Then $D$ is a free basis for a subgroup in $G^{\prime} * G^{\prime \prime}$.

THEOREM 2. Let $G^{\prime}$ and $G^{\prime \prime}$ be free $k_{\omega}$ groups. Then $D$ is a closed free topological basis for a closed subgroup $E$ in $G^{\prime} * G^{\prime \prime}$.

Definition 1. The homomorphism $\Psi: G \rightarrow G^{\prime} * G^{\prime \prime}$ is a co-monoid structure (c.m.s.) on $G$ if it satisfies the following:

(i) (co-associativity) $\left(1_{G} * \Psi\right) \Psi=\left(\Psi * 1_{G}\right) \Psi$, and

(ii) (co-units) $\left(C * 1_{G}\right) \Psi=1_{G}$ and $\left(1_{G} * C\right) \Psi=1_{G}$, where $C: G \rightarrow Q$ is the homomorphism into the topological group which consists of one element and where we identify $G$ with $G * Q$.

TheOREM 3. Let $G$ be a free topological group with basis $X$. Then the map $\Psi: X \rightarrow G^{\prime} * G^{\prime \prime}$ such that $\Psi(x)=j^{\prime}(x) j^{\prime \prime}(x)$, extends uniquely to a c.m.s. $\Psi$ on $G$. 
THEOREM 4. Let $\Psi$ be a c.m.s. on a $k_{\omega}$ group $G$. Then the set $X=\left\{g \in G \mid \Psi(g)=j^{\prime}(g) j^{\prime \prime}(g)\right\}$ with the subspace topology of $G$, is a free topological basis for $G$. The set $X$ will be called the corresponding basis for $\Psi$.

An immediate consequence of these theorems is the main result of this paper:

COROLLARY. If $G$ is a $k_{\omega}$ group then there is a one-to-one correspondence between the c.m.s. on it and its free bases.

The proof of Theorem 1 which is purely algebraic is given in $\$ 5$. The proofs of Theorems $2-4$ which are mainly topological are given next in $\$ 4$.

4. Proofs of Theorems 2-4. We fix $k_{\omega}$ decompositions for the groups involved. We may assume (see [8]) that the group $G$ of the beginning of $\$ 3$ has the following $k_{\omega}$ decomposition: $G=\bigcup_{i=1}^{\infty} G_{i}$, where $G_{i}=G_{i}^{-1}$ and $G_{i} G_{j} \subset G_{i+j}$. Let $G^{\prime}=\bigcup_{i=1}^{\infty} G_{i}^{\prime}$ and $G^{\prime \prime}=\bigcup_{i=1}^{\infty} G_{i}^{\prime \prime}$ be the corresponding $k_{\omega}$ decompositions given by $i^{\prime}$ and $i^{\prime \prime}$ (see $\$ 3$ ).

Proof of Theorem 2. We are required to prove that $E$ and $F(D)$ are isomorphic, and that $E$ is closed in $G^{\prime} * G^{\prime \prime}$. For this it will be convenient to have a $k_{\omega}$ structure on $F(D)$. This is achieved by showing first that $D$ is a closed subset of $G^{\prime} * G^{\prime \prime}$. The embedding of $G^{\prime} \times G^{\prime \prime}$ in $G^{\prime} * G^{\prime \prime}$ given by Theorem $\mathrm{C}(\mathrm{c})$, maps the diagonal of $G^{\prime} \times G^{\prime \prime}$, which is a closed subset, onto the set $D$. Thus it is enough to show that the image of $G^{\prime} \times G^{\prime \prime}$ in $G^{\prime} * G^{\prime \prime}$ under the embedding is also closed. Let $h$ be a map which appoints to each tuple in elements of $G^{\prime} \vee G^{\prime \prime}$ the corresponding reduced word in $G^{\prime} * G^{\prime \prime}$. By Theorem $\mathrm{C}(\mathrm{b})$ and Definition $\mathrm{A}(\mathrm{a})$ (iii), we have to show that $h\left(G^{\prime} \times G^{\prime \prime}\right) \cap h\left(\left(G_{i}^{\prime} \times G_{i}^{\prime \prime}\right)^{i}\right)$ is compact for all $i$. This follows from the equality:

$$
h\left(G^{\prime} \times G^{\prime \prime}\right) \cap h\left(\left(G_{i}^{\prime} \vee G_{i}^{\prime \prime}\right)^{i}\right)=h\left(\left(G_{i}^{\prime}\right)^{i}\right) \cup h\left(\left(G_{i}^{\prime}\right)^{i-1} \times G_{i}^{\prime \prime}\right) \cup \ldots \cup h\left(G_{i}^{\prime} \times\left(G_{i}^{\prime \prime}\right)^{i-1}\right) \cup h\left(\left(G_{i}^{\prime \prime}\right)^{i}\right),
$$

because each of the elements in the union is compact. We conclude that $D$ is a $k_{\omega}$ space and can be given the following $k_{\omega}$ decomposition: $D=\bigcup_{n=1}^{\infty} D_{n}$ where $D_{n}=D \cap h\left(\left(G_{n}^{\prime} \vee G_{n}^{\prime \prime}\right)^{n}\right)$. Now we are ready to complete the proof of the theorem. Since the group structure is clear it is sufficient to show that for any $k$ there is an $l$, both being integers, such that

$$
h\left(\left(G_{k}^{\prime} \vee G_{k}^{\prime \prime}\right)^{k}\right) \cap E \subset F_{l}\left(D_{l}\right) \text {. }
$$

Let $w \in h\left(\left(G_{k}^{\prime} \vee G_{k}^{\prime \prime}\right)^{k}\right) \cap E$. Then $w=g_{1} h_{2} g_{3} \ldots h_{t}$, where $g_{j} \in G_{k^{2}}^{\prime}, h_{j} \in G_{k^{2}}^{\prime \prime}$ and $t<k$. We can express $w$ in the following form:

$$
\begin{array}{r}
w=\left(g_{1} h_{1}\right)\left(h_{1}^{-1} h_{2} g_{3} h_{4} \ldots\right)=\left(g_{1} h_{1}\right)\left(h_{1}^{-1} h_{2} g_{1}^{-1} g_{2}\right)\left(g_{2}^{-1} g_{1} g_{3} h_{4} \ldots\right)=\ldots \\
=\left(g_{1} h_{1}\right)\left(h_{1}^{-1} h_{2} g_{1}^{-1} g_{2}\right) \ldots\left(g_{t-2}^{-1} g_{t-4}^{-1} \ldots g_{2}^{-1} g_{1} g_{3} \ldots g_{t-1} h_{t-2}^{-1} h_{t-4}^{-1} \ldots h_{2}^{-1} h_{1} h_{3} \ldots h_{t-1}\right) \\
\left(h_{t-1}^{-1} \ldots h_{3}^{-1} h_{1}^{-1} h_{2} \ldots h_{t-4} h_{t-2} h_{t}\right)
\end{array}
$$

where $\left(i^{\prime}\right)^{-1}\left(g_{j}\right)=\left(i^{\prime \prime}\right)^{-1}\left(h_{j}\right), i=1,2, \ldots, t$, and $g_{1}, h_{t}$ are possibly $e$.

The last equality is obtained by induction and the last bracket contains $e$, because $w \in E$. If we take $l$ to equal $k^{3}$ we get $w \in F_{l}\left(D_{l}\right)$.

Proof of Theorem 3. It is enough to show that $\Psi$ is a continuous map. Because if this is 
proved, from the universal property of free topological groups, $\Psi$ extends uniquely to a continuous homomorphism $\Psi: G \rightarrow G^{\prime} * G^{\prime \prime}$, which is easily seen to be a c.m.s. However the continuity of $\Psi$ follows from observing that $\Psi$ is the composition of the following continuous maps:

$$
X \stackrel{d}{\rightarrow} X \times X \stackrel{(i, i)}{\rightarrow} G \times G \stackrel{\left(j^{\prime}, j^{\prime \prime}\right)}{\longrightarrow}\left(G^{\prime} * G^{\prime \prime}\right) \times\left(G^{\prime} * G^{\prime \prime}\right) \stackrel{m}{\rightarrow} G^{\prime} * G^{\prime \prime},
$$

where $d$ is the diagonal map, $i$ the inclusion of $X$ in $G$, and $m$ the multiplication of $G^{\prime} * G^{\prime \prime}$.

Proof of Theorem 4. We start the proof by assigning to $X$ a $k_{\omega}$ decomposition $X=\bigcup_{n=1}^{\infty} X_{n}$ where $X_{n}=X \cap G_{n}$. This is possible if we show that $X$ is closed in $G$. However, by the proof of Theorem 2, $D$ is closed in $G^{\prime} * G^{\prime \prime}$ and since $X=\Psi^{-1}(D)$ the result follows. To complete the proof we have to show that $F(X)$ is isomorphic to $G$. To do this it suffices to show that for any $k$ there is an $l$ such that $G_{k} \subset F_{l}\left(X_{l}\right)$. Since $G_{k}$ is compact $\Psi\left(G_{k}\right) \subset F_{t}\left(D_{t}\right)$ for some integer $t$ by Theorem 2. Let $w \in G_{k}$. Then $\Psi(w)=d_{1} d_{2} \ldots d_{s}$ where $d_{j}=\left(g_{j} h_{j}\right)^{ \pm 1}$ for $g_{j} \in G_{t^{2}}^{\prime}, h_{j} \in G_{t^{2}}^{\prime \prime}$, $\left(i^{\prime}\right)^{-1}\left(g_{j}\right)=\left(i^{\prime \prime}\right)^{-1}\left(h_{j}\right)$ and $s \leqq t$. Since $X$ is the algebraic basis corresponding to $\Psi$ (Theorem A), we have $w=x_{1} \ldots x_{r}, x_{j} \in X \vee X^{-1}$ and $\Psi(w)=\Psi\left(x_{1}\right) \ldots \Psi\left(x_{r}\right)$ with $\Psi\left(x_{j}\right) \in D$. Because $D$ is free we conclude that $r=s, d_{j}=\Psi\left(x_{j}\right)$ and $g_{j}=x_{j} \in X_{t^{2}}$. Thus, $w \in F_{t^{2}}\left(X_{t^{2}}\right)$ and if $l=t^{2}$ we get $G_{k} \subset F_{l}\left(X_{l}\right)$.

5. Proof of Theorem 1. The proof is somewhat lengthy. We introduce some notation and definitions to state the theorem in a more convenient way.

Notations AND Definitions. (a) Let $F_{Y}$ and $F_{Z}$ denote the underlying groups of $F(Y)$ and $F(Z)$, respectively. Let $Z$ be a copy of $Y$ and $\theta: F_{Y} \rightarrow F_{Z}$ the identity isomorphism induced by the identity map from $Y$ to $Z$. For any $y_{i} \in \tilde{Y}\left(=Y \vee Y^{-1}\right)$, let $z_{i}=\theta\left(y_{i}\right)$.

(b) $J_{n}=\left\{y_{1} \ldots y_{n} z_{1} \ldots z_{n} \in F_{Y \vee Z}\right\}$, where $y_{1} \ldots y_{n}$ is a reduced word in elements of $Y$.

(c) $K_{n}=\bigcup_{i=1}^{n} J_{i}$.

(d) $K=\bigcup_{n=1}^{\infty} K_{n}$.

(e) For any $w \in F_{\mathrm{Y} \vee Z}, l(w)$ will denote the length of the reduced word in elements of $Y \vee Z$.

(f) Since an element of $K$ is a word in elements of $Y \vee Z$, a word $u$ in elements of $K$ is also a word in elements of $Y \vee Z$. Thus, its length is well defined. Let $u=v_{1} \ldots v_{n}, v_{i} \neq v_{i+1}^{-1}$, $i=1, \ldots, n-1$, be a word in elements of $K$. Then $u$ is called a simple word if $l(u)=\max _{1 \leqq i \leqq n}\left\{l\left(v_{i}\right)\right\}$.

Since the free product of the free groups $F_{Y}$ and $F_{Z}$ is the free group $F_{Y \vee Z}$, Theorem 1 can be stated as follows:

RESTATEMENT OF THEOREM $1 . K$ is a free subset of $F_{Y \vee Z}$.

This will be proved by the aid of the following propositions.

Proposition 1. Let $u^{1} \ldots, u^{n}$ be simple words in elements of $K$, such that $l\left(u^{i} u^{l+1}\right)>l\left(u^{l}\right)$, $l\left(u^{i+1}\right), 1 \leqq i \leqq n-1$. Then $l\left(u^{1} \ldots u^{n}\right)>l\left(u^{1}\right), \ldots, l\left(u^{n}\right)$. 
Proposition 2. Let $u=v_{1} \ldots v_{q}, v_{i} \neq v_{i+1}^{-1}, 1 \leqq i \leqq q-1$, be a word in elements of $K$. Then its factors can be bracketed so that $u=u^{1} \ldots u^{k}$, where $u^{i}$ are simple words and $l\left(u^{i} u^{i+1}\right)>l\left(u^{i}\right)$, $l\left(u^{i+1}\right), 1 \leqq i \leqq k-1$.

The proofs of the propositions are transferred to the end of this section.

Proof of Theorem 1. Let $u=v_{1} \ldots v_{n}, v_{i} \neq v_{i+1}^{-1}, i=1, \ldots, n-1$, be a word in elements of $K$. To prove the theorem it is enough to show that $l(u)>1$, and thus $K$ is free. By Proposition 2 we can bracket $u$ into simple words $u=u^{1} \ldots u^{k}$, such that $l\left(u^{i} u^{i+1}\right)>l\left(u^{i}\right), l\left(u^{i+1}\right), 1 \leqq i \leqq$ $k-1$. Now $u^{1}, \ldots, u^{k}$ satisfy the conditions of Proposition 1 , and since $l\left(u^{i}\right) \geqq 2,1 \leqq i \leqq k$, we get that $l(u)>1$.

Proof of Proposition 1. The proof is by induction on the number of the simple words. Suppose $n=2, l\left(u^{1}\right)=2 a, l\left(u^{2}\right)=2 b$ and $c=\min \{a, b\}$. In the reduced form of $u^{1} u^{2}$ at most $2(c-1)$ elements are cancelled out, for if more elements are cancelled out it contradicts the fact that $l\left(u^{1} u^{2}\right)>l\left(u^{1}\right), l\left(u^{2}\right)$. Furthermore, the first $a+1$ elements and the last $b+1$ elements of $u^{1} u^{2}$ are the first $a+1$ elements of $u^{1}$ and the last $b+1$ elements of $u^{2}$. We conclude that the last $b+1$ elements of $u^{1} u^{2}$ equal the last $b+1$ elements of $u^{2}$ and that $l\left(u^{1} u^{2}\right)>l\left(u^{1}\right), l\left(u^{2}\right)$. We now assume that the conclusion holds for $n \geqq 2$, and consider the case for $n+1$. Let $l\left(u^{n}\right)=2 d, l\left(u^{n+1}\right)=2 f$ and $g=\min \{d, f\}$. As in the case for $n=2$, at most $2(g-1)$ elements are cancelled out in the reduced form of $u^{n} u^{n+1}$, leaving the first $d+1$ elements of $u^{n}$ and the last $f+1$ elements of $u^{n+1}$ unaffected. As a consequence we get $l\left(u^{1} \ldots u^{n}\right)<l\left(u^{1} \ldots u^{n+1}\right)$. We now consider two cases.

(i) $g=d$. In this case $l\left(u^{1} \ldots u^{n+1}\right) \geqq l\left(u^{1} \ldots u^{n}\right)-2(d-1)+l\left(u^{n+1}\right)>l\left(u^{n+1}\right), l\left(u^{1} \ldots\right.$ $\left.u^{n+1}\right)>l\left(u^{1} \ldots u^{n}\right)>l\left(u^{i}\right), 1 \leqq i \leqq n$, which follows from the induction assumption and the previous mentioned consequence.

(ii) $g=f$. Because of the previous mentioned consequence and the induction assumption $l\left(u^{i}\right)<l\left(u^{1} \ldots u^{n}\right)<l\left(u^{1} \ldots u^{n+1}\right), \quad 1 \leqq i \leqq n$. However, since $l\left(u^{n+1}\right) \leqq l\left(u^{n}\right)$ we have $l\left(u^{n+1}\right) \leqq l\left(u^{n}\right)<l\left(u^{1} \ldots u^{n+1}\right)$. This completes the induction step and the proposition is proved.

Proof of Proposition 2. The proof is by induction on the sets $K_{n}$. The proposition is trivial for $n=1$. We assume that it is correct for $n$ and prove it for $n+1$. This will be done via two lemmas which will be proved under the induction assumption.

LemMa 1. Let $v_{1} \ldots v_{k}$ be a simple word in elements of $K_{n}$. Then every word of the form $v_{1} \ldots v_{s}$ or $v_{s} \ldots v_{k}, 1<s<k$, is also simple.

Proof. We prove the lemma only for words of the first form. Words of the second form can be treated analogously. Suppose the lemma is not correct. Then there exists $s$ such that $v_{1} \ldots v_{s}$ is not simple, while $v_{1} \ldots v_{s+1}$ is simple. By the induction assumption we have: $v_{1} \ldots v_{s}=u^{1} \ldots u^{r}, r>1$, where $u^{i}$ are simple words and $l\left(u^{i} u^{i+1}\right)>l\left(u^{i}\right), l\left(u^{i+1}\right), 1 \leqq i \leqq r-1$. We consider several possibilities.

(i) $l\left(u^{r} v_{s+1}\right)>l\left(u^{r}\right), l\left(v_{s+1}\right)$. Since $v_{s+1}$ is a simple word Proposition 1 can be applied and we obtain that $l\left(u^{1} \ldots u^{r} v_{s+1}\right)>l\left(u^{1}\right), \ldots, l\left(u^{r}\right)$, or $l\left(v_{1} \ldots v_{s+1}\right)>l\left(v_{1}\right), \ldots, l\left(v_{s+1}\right)$, which contradicts the fact that $v_{1} \ldots v_{s+1}$ is a simple word. 
(ii) $l\left(u^{r} v_{s+1}\right)=\max \left\{l\left(u^{r}\right), l\left(v_{s+1}\right)\right\}$. In this case $u^{r} u_{s+1}$ is a simple word and Proposition 1 applies to $u^{1}, \ldots, u^{r-1}, u^{r} v_{s+1}$. Thus, $l\left(u^{1} \ldots u^{r} v_{s+1}\right)>l\left(u^{1}\right), \ldots, l\left(u^{r-1}\right), l\left(u^{r} v_{s+1}\right)$ or $l\left(v_{1} \ldots v_{s+1}\right)>l\left(v_{1}\right), \ldots, l\left(v_{s+1}\right)$, which is again a contradiction to the fact that $v_{1} \ldots v_{s+1}$ is simple.

(iii) $l\left(u^{r} v_{s+1}\right)<\max \left\{l\left(u^{r}\right), l\left(v_{s+1}\right)\right\}$.

In this case $l\left(u^{r} v_{s+1}\right)$ is smaller than the length of the element of $K_{n}$ in $u^{r} v_{s+1}$ with maximal length. By the induction assumption the elements of $K_{n}$ in $u^{r} v_{s+1}$ can be bracketed so to fulfil the conditions of Proposition 1. The application of Proposition 1 leads to a contradiction. These contradictions prove the lemma.

LEMMA 2. Every simple word in elements of $K_{n}$ has only one element, say $v$, of $K_{n}$ with maximal length. The central two elements of the simple word equal the central two elements of $v$.

Proof. Throughout the proof we use the previous lemma without mentioning it explicitly. To prove the first part of the lemma it is enough to obtain a contradiction to the assumption that the simple word consists of two elements of maximal length and elements of smaller length in between. Let this simple word be $v_{1} \ldots v_{m}$ where $v_{1}=y_{1} \ldots y_{p} z_{1} \ldots z_{p}$. Since $v_{1} v_{2}$ is a simple word, $v_{2}$ must be of the form $z_{p}^{-1} \ldots z_{p-a+1}^{-1} y_{p}^{-1} \ldots y_{p-a+1}^{-1}$ with $0<a<p$ and $v_{1} v_{2}=y_{1} \ldots y_{p} \ldots y_{p}^{-1} \ldots y_{p-a+1}^{-1}$. Assume that for $i \geqq 2, l\left(v_{i-1}\right)>l\left(v_{i}\right)$, and $v_{1} \ldots v_{i}=$ $y_{1} \ldots y_{p} \ldots z_{l} \ldots z_{l+b-1}$ or $v_{1} \ldots v_{i}=y_{1} \ldots y_{p} \ldots y_{l}^{-1} \ldots y_{l-b+1}^{-1}$ where $l\left(v_{i}\right)=2 b$. We now prove the induction step. Let the first of the possibilities in the induction assumption hold (the other possibility can be treated analogously). Since $v_{1} \ldots v_{i+1}$ is a simple word, $v_{i+1}$ must be of the form $v_{i+1}=z_{l+b-1}^{-1} \ldots z_{l+b-c}^{-1} y_{l+b-1}^{-1} \ldots y_{l+b-c}^{-1}, 0<c<b$, and we get $l\left(v_{i}\right)>l\left(v_{i+1}\right)$, $v_{1} \ldots v_{i+1}=y_{1} \ldots y_{p} \ldots y_{l+b-1}^{-1} \ldots y_{l+b-c}^{-1}$. This completes the induction and we conclude that $l\left(v_{1}\right)>l\left(v_{2}\right)>\ldots>l\left(v_{m}\right)$ or $l\left(v_{1}\right)>l\left(v_{m}\right)$, which is a contradiction. The proof of the second part of the lemma follows easily from the proof of the first part.

Completion of the proof of Proposition 2. Let $w$ be a word in elements of $K_{n+1}$. We can express $w$ as a product of words in $K_{n}$ and words in $J_{n+1}$ so that the two kinds of words alternate. Every one of the words in $K_{n}$ can be bracketed to satisfy the result of the proposition by the induction assumption. As for the words in $J_{n+1}$ their elements already satisfy the result of the proposition, because each element is simple and if $v_{1}, v_{2} \in J_{n+1}, v_{1} \neq v_{2}^{-1}$, then $l\left(v_{1} v_{2}\right)>$ $l\left(v_{1}\right), l\left(v_{2}\right)$. Let $w=u^{1} \ldots u^{s}$ be the word with the bracketing mentioned above. We prove that we can add some new brackets and obtain the result of the proposition. The proof is by induction on the number of the simple words. For $k=1$ there is nothing to prove. We assume that the proposition holds for $k \geqq 1$ and prove that it holds also for $k+1$. Suppose that brackets were introduced in $u^{1} \ldots u^{k}$ to obtain $a^{1} \ldots a^{l}$ with the properties of the proposition. We prove that this can be done also for $u^{1} \ldots u^{i+1}$. Several cases are to be considered:

(i) $u^{k}, u^{k+1}$ are both words in elements of $K_{n}$ or $u^{k}, u^{k+1} \in J_{n+1}$. The required form is $a^{1} \ldots a^{l} u^{k+1}$.

(ii) $u^{k}$ is a word in elements of $K_{n}, u^{k+1} \in J_{n+1}$ and $l\left(u^{k} u^{k+1}\right)>l\left(u^{k}\right), l\left(u^{k+1}\right)$. The required form is $a^{1} \ldots a^{l} u^{k+1}$. (The same holds if $u^{k}$ and $u^{k+1}$ reverse roles.)

(iii) $u^{k}$ is a word in elements of $K_{n}, u^{k+1} \in J_{n+1}$ and $l\left(u^{k} u^{k+1}\right)=l\left(u^{k+1}\right)$. We introduce one 
bracket which turns $a^{l}$ and $u^{k+1}$ into a simple word, and the result $a^{1} \ldots a^{l-1}\left(a^{l} u^{k+1}\right)$ has the required properties. (The same holds if $u^{k}$ and $u^{k+1}$ reverse roles.)

(iv) $u^{k}$ is a word in elements of $K_{n}, u^{k+1} \in J_{n+1}$ and $l\left(u^{k} u^{k+1}\right)<l\left(u^{k+1}\right)$. We prove that this case is impossible. By Lemma 2 the central two elements of $u^{k}$ equal the central two elements of an element in $K_{n}$. Thus one of them belongs to $\widetilde{Y}$ and the other to $\tilde{Z}$. Since $l\left(u^{k} u^{k+1}\right)<$ $l\left(u^{k+1}\right)$ those two elements must get cancelled out by the first $n+1$ elements of $u^{k+1}$. But all the first $n+1$ elements of $u^{k+1}$ belong to $\tilde{Y}$ or to $\tilde{Z}$, and thus they cannot cancel out the above specified two elements. This produces a contradiction. (The same holds if $u^{k}$ and $u^{k+1}$ reverse roles.) This completes the induction step and proves the proposition.

6. Applications. (a) Theorems 3 and 4 give us many c.m.s. on a group once one is given. This follows from the remark in [2, p. 23] that from a given topological free basis of a group we can construct many bases. There it was done for disconnected bases. However, this procedure extends also to connected spaces of the following form. Let $X_{1}$ and $X_{2}$ be compact sets such that $X_{1} \cap X_{2}=e$. Then $X_{1} \cup a_{1} X_{2}$ is a basis of $F\left(X_{1} \vee X_{2}\right)$ for any $a_{1} \in X_{1}$.

(b) We demonstrate how to use a c.m.s. to prove a statement about free bases. This is done for a theorem in [2, pp. 38-41].

THEOREM. Let $X$ and $B$ be free topological bases of $G$. If $X$ is compact so is $B$.

Proof. Assume $B$ is not compact. There then exists a sequence $\left\{b_{n}\right\} \subset B$ such that the length of $\left\{b_{n}\right\}$ in elements of $X$ is unbounded. Define the sequence $\left\{x_{n}\right\} \subset X$ as follows: $x_{n}$ is a product of $b_{n}$ and other elements of $B$. This is possible since both $X$ and $B$ are bases. Let $\Phi$ be a c.m.s. corresponding to $B$. Then by Propositions 1 and 2 we conclude that the length in elements of $Y \vee Z$, of the elements in the sequence $\left\{\Phi\left(x_{n}\right)\right\}$ is unbounded. By standard procedure, we can extract an infinite subset of $\left\{\Phi\left(x_{n}\right)\right\}$ which is a discrete set. The corresponding subset $\left\{\bar{x}_{n}\right\}$ is also a discrete infinite subset of $X$, because $\Phi$ is a monomorphism. This contradicts the fact that $X$ is compact.

(c) We would like to remark that once a group has a c.m.s. on it, its homology structure is known.

THEOREM. Let the group $G$ of Theorem 4 be connected. Then the homology of $G$ over a field is a connected free tensor algebra.

Proof. Theorem 4 provides $G$ with a subset $X$, so that $G$ is topologically isomorphic to $F(X)$. Define $r^{\prime}: X \rightarrow F(X)$ so that $r^{\prime}(x)=x^{-1}$ for $x \in X$. This function is obviously continuous. Let $r: F(X) \rightarrow F(X)$ be the unique extension of $r^{\prime}$. This map is called the inverse function for the c.m.s., and turns $G$ into a co-group (see [1]). Since the homology functor with field coefficients from TG to the category of connected graded algebras (C.G.A.) preserves coproducts, it turns $G$ into a co-group in the category of C.G.A. Thus Theorem 1.2 of [1] can be applied and the theorem follows at once.

\section{REFERENCES}

1. I. Berstein, On Co-groups in the category of graded algebras, Trans. Amer. Math. Soc. 115 (1965), 257-269. 
2. M. I. Graev, Free topological groups (Russian), Izv. Akad. Nauk SSSR. Ser. Mat. 12 (1948), 279-324. (English Transl. Amer. Math. Soc. Transl. (1). 8 (1951), 305-364.)

3. M. I. Graev, On free products of topological groups (Russian), Izv. Akad. Nauk. SSSR. Ser. Mat. 14 (1950), 343-354.

4. P. J. Hilton, Homotopy and duality (Gordon and Breach, 1965).

5. D. M. Kan, On monoids and their dual, Bol. Soc. Mat. Mexicana 3 (1958), 52-61.

6. J. Mack, S. A. Morris and E. T. Ordman, Free topological groups and the projective dimension of a locally compact abelian group, Proc. Amer. Math. Soc. 40 (1973), 303-308.

7. S. A. Morris and E. T. Ordman, The topology of free products of topological groups, Lecture Notes in Mathematics 372 (1974), 504-513.

8. E. T. Ordman, Free products of topological groups which are $k_{\omega}$-spaces Trans. Amer. Math. Soc. 191 (1974), 61-73.

Department of Mathematics

HAIFA UNIVERSITY

HAIFA

ISRAEL 